Proc. of the XI Int. Conf. - Ion Implantation and other Applications of Ions and Electrons, Kazimierz Dolny 2016

\title{
Mechanical Properties of the Stellite 6 Cobalt Alloy Implanted with Nitrogen Ions
}

\author{
P. Budzyński ${ }^{a, *}$, M. Kamiński ${ }^{a}$, M. Wiertel $^{b}$, K. Pyszniak $^{b}$ And A. Droździel ${ }^{b}$ \\ ${ }^{a}$ Faculty of Mechanical Engineering, Lublin University of Technology, Nadbystrzycka 36, 20-618 Lublin, Poland \\ ${ }^{b}$ Institute of Physics, M. Curie-Skłodowska University, pl. M. Curie-Skłodowskiej 1, 20-031 Lublin, Poland \\ The effect of nitrogen ion implantation on Stellite 6 cobalt alloy was investigated. In this research, cobalt alloy \\ was implanted with $65 \mathrm{keV}$ nitrogen ions at the fluence of $(1 \div 10) \times 10^{16} \mathrm{~N}^{+} / \mathrm{cm}^{2}$. The distribution of implanted \\ nitrogen ions and vacancies produced by them was calculated using the SRIM program. The surface morphology \\ was examined and the elemental analysis was performed using scanning electron microscopy, energy dispersive \\ $\mathrm{X}$-ray spectroscopy and grazing incidence X-ray diffraction. The wear tests were conducted with the use of the \\ pin-on-disc method. The results demonstrate that implantation with nitrogen ions significantly reduces the friction \\ factor and wear. The friction coefficient of the implanted sample at the fluence of $1 \times 10^{17} \mathrm{~N}^{+} / \mathrm{cm}^{2}$ increased to \\ the values characteristic of an unimplanted sample after 5000 measurement cycles. The depth of the worn trace \\ was about $2.0 \mu \mathrm{m}$. This implies that the thickness of the layer modified by the implantation process is $\approx 2.0 \mu \mathrm{m}$ \\ and exceeds the initial range of the implanted ions by an order of magnitude. This is referred to as a long-range \\ implantation effect. The investigations have shown that the long-range effect is caused by movement of not only \\ implanted nitrogen atoms but also carbon dopant atoms towards the friction zone. Diffusion of carbon atoms has \\ been documented here for the first time. Furthermore, the increased content of oxygen atoms on the track bottom \\ indicates a dominant oxidative wear of the Stellite samples after nitrogen implantation with the energy $65 \mathrm{keV}$ and \\ the fluences of $5 \times 10^{16}$ and $10^{17} \mathrm{~N}^{+} / \mathrm{cm}^{2}$. \\ DOI: 10.12693 /APhysPolA.132.203 \\ PACS/topics: 68.55.Ln, 62.20.Qp, 61.82.Bg, 61.80.--x
}

\section{Introduction}

Stellite is a range of alloys of cobalt with chromium, tungsten, carbon, and other elements. Microphotographs of the structure and metallurgically polished sections of these alloys exhibit visible star-like areas (stella — Latin name of a star), hence their name stellites. The alloys are abrasion- and organic acid-resistant and heatproof. Stellites are widely applied in aviation, space, and automotive technology and in biomedical engineering as implant material. They are used for production of high-quality tools, parts of combustion engines, and other elements operating in extreme heat conditions [1]. There are investigations aimed at improvement thereof and expansion of their applications [2]. Nitrogen implantation is used for improvement of the mechanical properties of steel [3-6], aluminium [7], Ti6Al4V alloy [8] etc. Nitrogen implantation is also applied for improvement of the mechanical properties of sensitive elements in mechanical structures. Motorisation industry requires materials for valves and valve faces in internal combustion engines, for instance a Stellite. In publication [9], the effect of nitrogen ion implantation with energy of $65 \mathrm{keV}$ on the microhardness of the Stellite 6 alloy was investigated. Implantation with a dose of $5 \times 10^{16} \mathrm{~N}^{+} / \mathrm{cm}^{2}$ increases alloy microhardness by $20 \%$. It was interesting to explore the influence of nitrogen implantation on the coefficient of friction and alloy wear as well as the occurrence and causes of the long-range effect.

*corresponding author; e-mail: p.budzynski@pollub.pl

\section{Materials and methods}

The investigations were carried out on a commercial alloy Stellite 6. It contains Cr 30\%, W 4.5\%, C 1.2\%, Ni $2 \%$, and $\mathrm{Fe} 2 \%$; cobalt accounts for $60.3 \%$. Nitrogen implantation was performed at the energy $65 \mathrm{keV}$. The fluence of the implanted ions was $10^{16}, 5 \times 10^{16}$, and $10^{17} \mathrm{~N}^{+} / \mathrm{cm}^{2}$. The distribution of the implanted ions and point defects (gaps) at equal sample depths was calculated with the use of SRIM programs [10]. The measurements of the friction and wear coefficients were carried out in a tribological test under the conditions of technically dry friction on a custom-made pin/ball-on disc tribometer [11] and on an Antonpaar Nano-Tribometer NTR2 (CFM Instrument). A tungsten carbide ball with a diameter of $=0.5 \mathrm{~mm}$ and $500 \mathrm{mN}$ contact force was used as a counter-sample.

Next the wear trace was measured using the Taylor Hobson Form Talysurf $50 \mathrm{~mm}$ Intra profile measurement gauge. The measurements provided profilograms enabling determination of the mean wear of wear trace. The wear was defined as the mean surface of the crosssection of contact between the sample and the countersample. Changes in the surface layer were analysed with the grazing incidence X-ray diffraction (GXRD) method. The measurements were carried out using an Empyrean X-ray diffractometer from PANalytical equipped with a copper lamp. The gloss angle was $2^{\circ}$.

\section{Results}

For the purpose of preliminary determination of ionimplantation, a numerical simulation was performed. Typically, numerical simulations correspond well with the experimental results. Figure 1 presents the calculated 
(predicted) distribution of nitrogen ions implanted at the energy $E=65 \mathrm{keV}$ and the fluence of $D=10^{17} \mathrm{~N}^{+} / \mathrm{cm}^{2}$ into a cobalt alloy and gaps generated thereby. It is evident that the calculated range of the implanted nitrogen ions that does not exceed $0.17 \mu \mathrm{m}$, and the maximum concentration of gaps is noted at a depth of $\approx 0.05 \mu \mathrm{m}$.

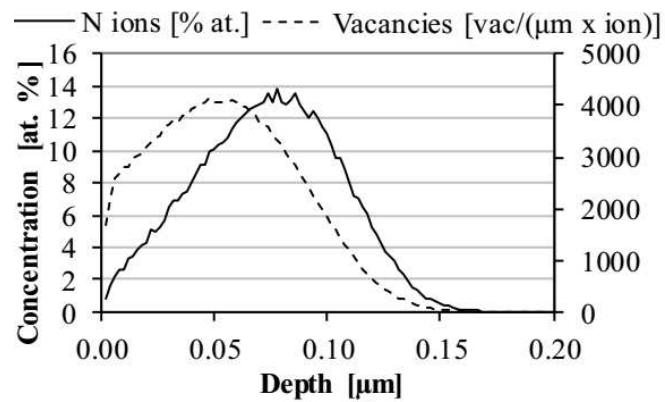

Fig. 1. Calculated distribution of nitrogen ions implanted at the energy $E=65 \mathrm{keV}$ and the fluence of $D=10^{17} \mathrm{~N}^{+} / \mathrm{cm}^{2}$ into the Stellite 6 cobalt alloy and gaps generated thereby.

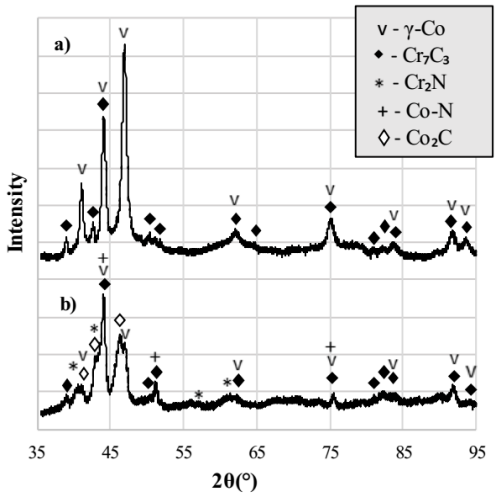

Fig. 2. GXRD spectrum of the Stellite 6 sample: (a) before and (b) after nitrogen implantation at energy $65 \mathrm{keV}$ and the fluence of $10^{17} \mathrm{~N}^{+} / \mathrm{cm}^{2}$.

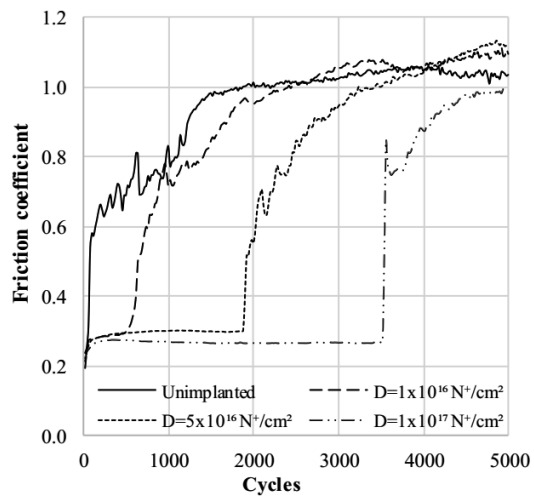

Fig. 3. Results of measurements of the friction coefficient for the Stellite 6 sample before and after implantation of nitrogen ions.

The results of changes in the surface layer after the ion implantation are shown in Fig. 2b. Figure 2a presents the sample surface structure before ion implantation.
The spectrum is relatively complex and not all peaks were identified. Nitrogen implantation induces formation of $\mathrm{Cr}_{2} \mathrm{~N}$ and $\mathrm{Co}-\mathrm{N}$ compounds. Furthermore, the implanted nitrogen ions form a compound $\mathrm{Co}_{2} \mathrm{C}$ with carbon, the content of which in the sample is $c a .1 .2 \%$.

The results of the measurements of the friction coefficient are shown in Fig. 3. Nitrogen implantation leads to reduction of the friction coefficient of the sample surface layer modified in the implantation process.

As can be seen, the friction coefficient for the implanted samples is lower than that for the unimplanted one noted during the first 2200, 3200, and 5000 cycles. This indicates that the thickness of the layer modified by the nitrogen implantation process at the fluence of $10^{17} \mathrm{~N}^{+} / \mathrm{cm}^{2}$ is $\approx 2.0 \mu \mathrm{m}$ and exceeds the initial range of the implanted ions by an order of magnitude. This effect, known as the long-range implantation effect, was first documented in paper [6] during a tribological test.

This implies that the thickness of the layer modified by the implantation process can be determined on the basis of the depth of the track generated during a test in which the friction coefficient was lower than that for the unimplanted sample. The test for the implanted samples was terminated when the friction coefficient reached the value that was characteristic of an unimplanted sample, i.e. $\mu \approx 1.0$. The cross-section of the track was measured with the use of a profilometer - Fig. 4.

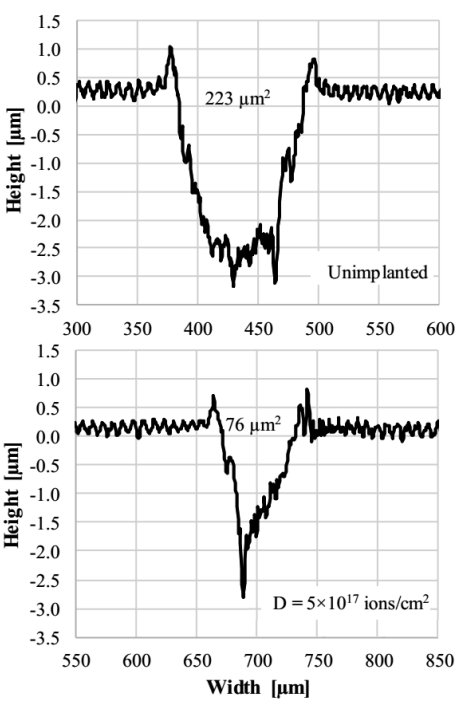

Fig. 4. Profilogram of a track worn by the ball on the Stellite 6 sample in the 5000 cycles test.

The mean value of the cross-sectional area of the track calculated from 20 measured profilograms was adopted as the measure of wear. The results are shown in Fig. 5. The wear declines with the increase of implanted fluence. After the implantation of the fluence of $10^{17} \mathrm{~N}^{+} / \mathrm{cm}^{2}$, there is a 3 -fold decrease in the wear value, compared with the unimplanted sample. One of the wear factors is the adhesive wear, which is evidenced by the peaks at the edges of the profilograms - Fig. 4 and the microphotograph - Fig. 6a. 


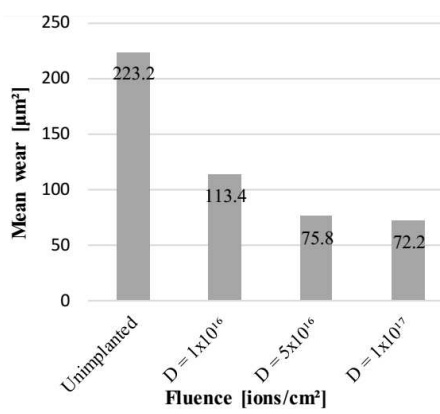

Fig. 5. Mean sample wear after 5000 measurements cycles.

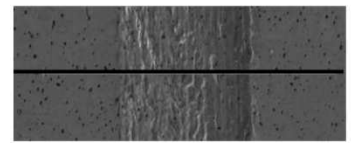

O K series
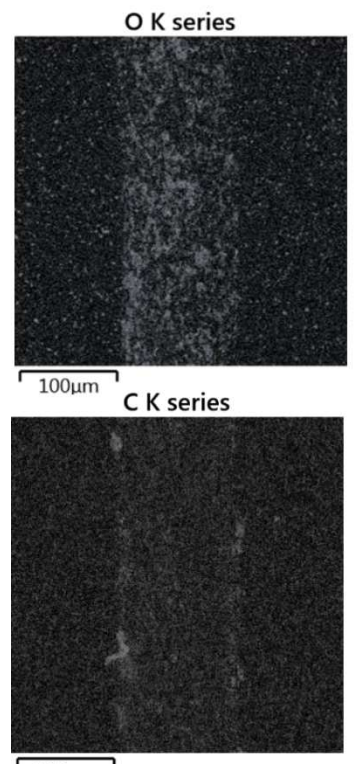

$\longdiv { 1 0 0 \mu m }$

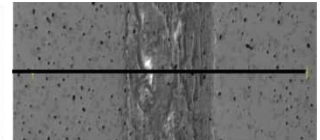

O K series

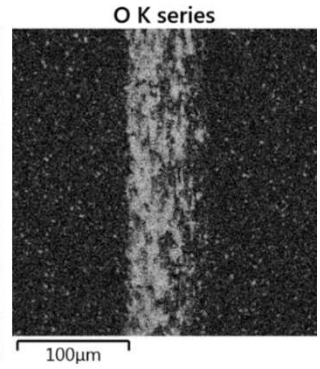

C K series

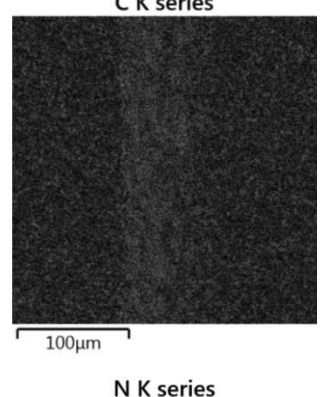

N K series

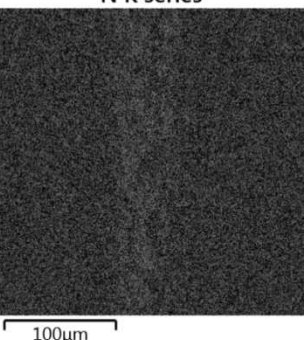

Fig. 6. Microphotograph of a track fragment worn on the Stellite 6 sample with a marked scan line and distribution of carbon, nitrogen, and oxygen atoms: (a) data for the unimplanted sample, (b) data for the sample implanted at the fluence of $10^{17} \mathrm{~N}^{+} / \mathrm{cm}^{2}$.

Changes in the contents of oxygen, carbon, and nitrogen atoms, which play an important role in the friction process, were examined with the energy dispersive X-ray spectroscopy (EDS) method. A scanning electron microscope TESCAN Vega 3LMU coupled with an EDX microanalyser Oxford Instruments AZtecEnergy-Advanced was used.
The analysis of the distribution of oxygen, carbon, and nitrogen atoms on the sample surface and track bottom was carried out with the EDS method. As shown in Fig. 6a,b, the contents of nitrogen, carbon, and oxygen on the bottom of the track are slightly higher than on the sample surface. The higher oxygen content suggests contribution of oxidative wear besides adhesive wear. The higher carbon and nitrogen contents on the bottom of the track than that on the sample surface indicate diffusion of carbon and nitrogen atoms into the friction zone caused by a local temperature rise occurring during the tribological test. Radiation damage may cause additional acceleration of diffusion.

\section{Summary}

The friction coefficient in the layer modified by the implantation process declined to the value of $\approx 0.3$, whereas it was $\approx 1.0$ for the unimplanted sample. Wear is also substantially reduced. The wear of the sample implanted at the fluence of $10^{17} \mathrm{~N}^{+} / \mathrm{cm}^{2}$ decreases 3-fold, compared with the unimplanted one. The improvement of the tribological properties of nitrogen-implanted samples is an effect of e.g. formation of the following compounds: $\mathrm{Cr}_{2} \mathrm{~N}, \mathrm{Co}_{2} \mathrm{C}$, and $\mathrm{Cr}-\mathrm{N}$. The reported research has shown that the long-range effect is caused by diffusion of not only implanted nitrogen atoms but also carbon atoms in the friction zone. The diffusion of carbon atoms has been demonstrated for the first time.

\section{Acknowledgments}

The authors express their gratitude to Dr Z. Surowiec for assistance in the X-ray diffraction acquisition.

\section{References}

[1] Y. Birol, Trans. Nonferrous Met. Soc. China 20, 1656 (2010).

[2] H.Ö. Gülsoy, Ö. Özgün, S. Bilketay, Mater. Sci. Eng. A 651, 914 (2016).

[3] J. Narojczyk, Z. Werner, J. Piekoszewski, W. Szymczyk, Vacuum 78, 229 (2005).

[4] P. Budzynski, L. Kara, T. Küçükömeroglu, M. Kaminski, Vacuum 122, 230 (2015).

[5] A.A. Youssef, P. Budzynski, J. Filiks, A.P. Kobzev, J. Sielanko, Vacuum 77, 37 (2004).

[6] P. Budzynski, Nucl. Instrum. Methods Phys. Res. B 342, 1 (2015).

[7] A.A. Youssef, P. Budzynski, J. Filiks, Z. Surowiec, Vacuum 78, 599 (2005).

[8] P. Budzynski, A.A. Youssef, J. Sielanko, Wear 261, 1271 (2006).

[9] P. Budzyński, M. Kamiński, K. Pałka, A. Droździel, M. Wiertel, IOP Conf. Series Mater. Sci. Eng. 148, 012046 (2016).

[10] J.F. Ziegler, Nucl. Instrum. Methods Phys. Res. B 219, 1027 (2004).

[11] P. Budzyński, P. Tarkowski, P. Penkala, Vacuum 63 731 (2001). 